(a)

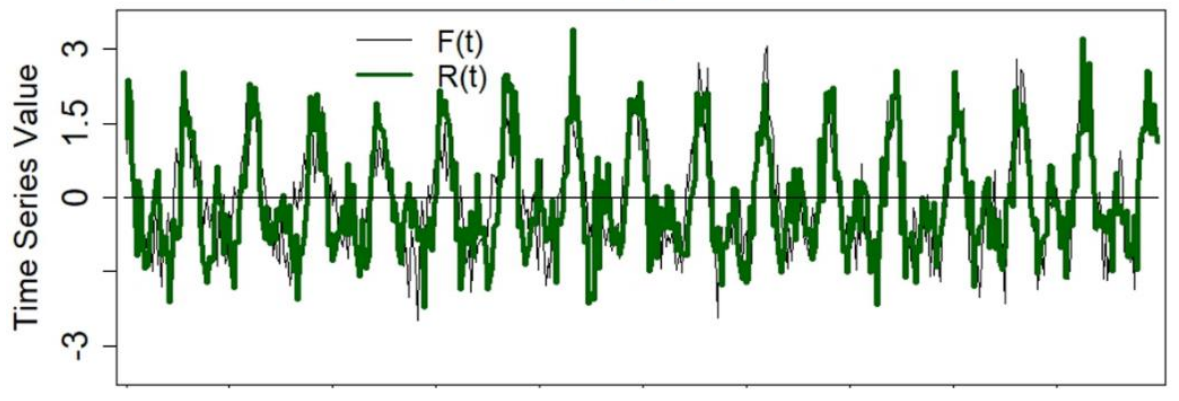

(b) Wavelet Coherence

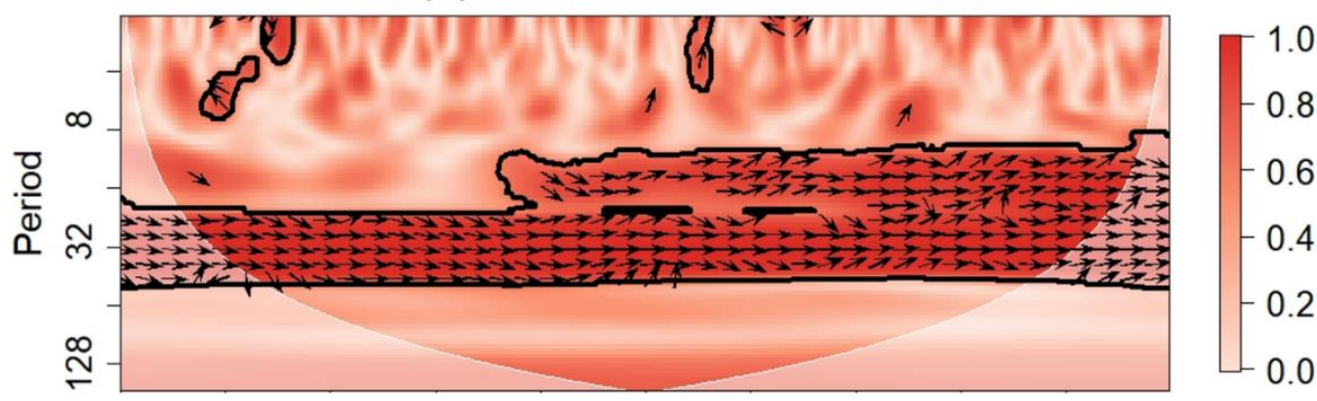

(c) Nonlinear Coherence

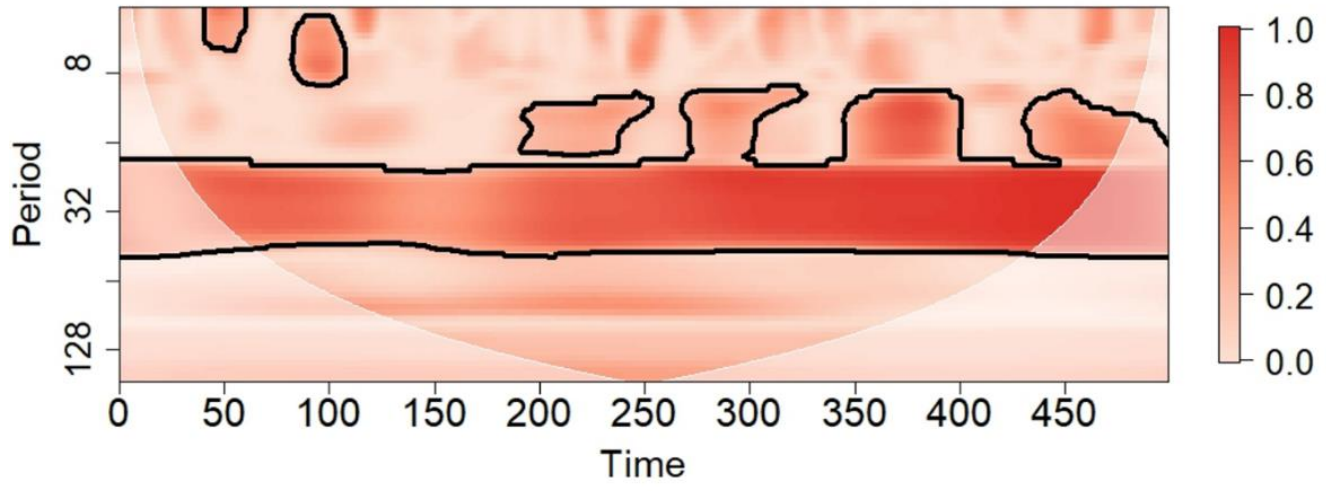

Figure S1. The forcing times series, $F(t)$, from the main text and the response $R(t)=\cos \left(\frac{2 \pi}{p_{1}} t+\phi\right)+\cos \left(\frac{2 \pi}{p_{3}}\right.$ t) $+W_{R}(t)$ where $p_{1}=32, p_{3}=p_{1} / 2=16$. (b) Linear coherence and (c) nonlinear coherence between $F(t)$ and $R(t)$. Contours enclose regions of $5 \%$ cumulative area-wise significance and the light-shaded region represents the cone of influence. Although linear coherence suggests that $F(t)$ and $R(t)$ are not related at the period $p_{3}=16$ from $t=0$ to almost $t=200$, nonlinear coherence shows that they are related, which should be the case by construction because both $R(t)$ and $F(t)$ have cosine functions with period of 16 . The time series $F(t)$ and $R(t)$ must be related at $p_{3}=16$ otherwise the relative bi-phase would fluctuate randomly according to Eq. (16), resulting in statistically insignificant nonlinear wavelet coherence at $p_{3}=$ 32. In other words, $\phi_{n}^{X}(32)-\phi_{n}^{Y}(32)$ and $\phi_{n}^{X}(16)-\phi_{n}^{Y}(16)$ do not fluctuate randomly so that $K$ is not a random function of time. 


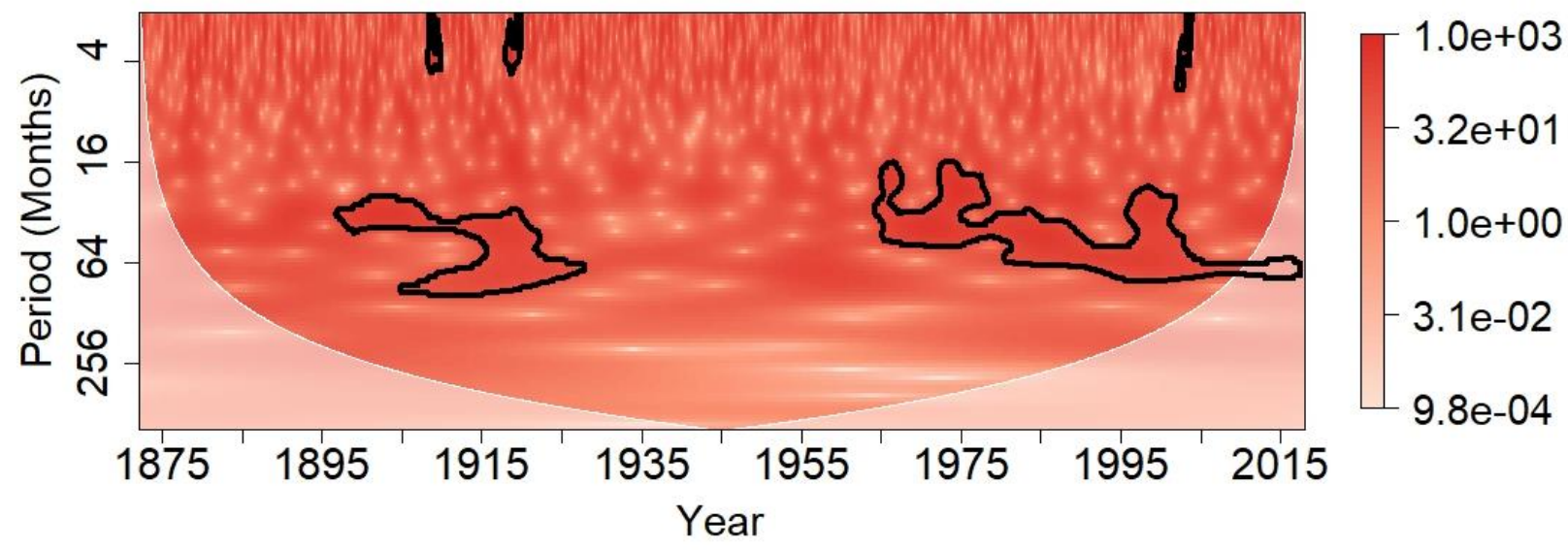

Figure S2. Wavelet power spectrum of Nino 1+2 - AIR. 
(a) All India

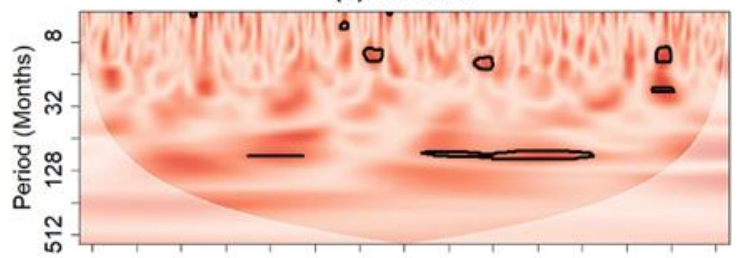

(c) Northwest

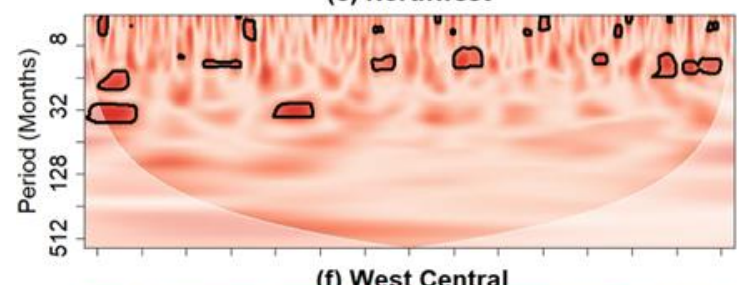

(f) West Central

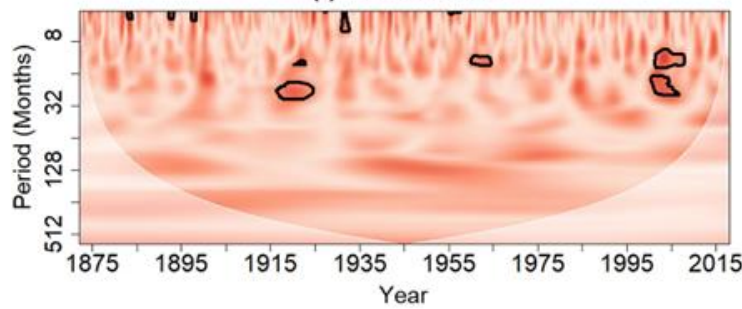

(b) Peninsula

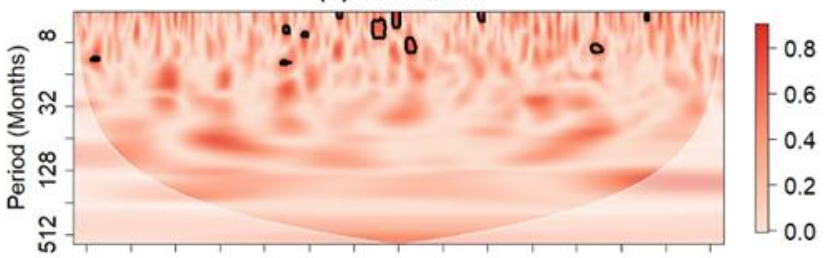

(d) Northeast

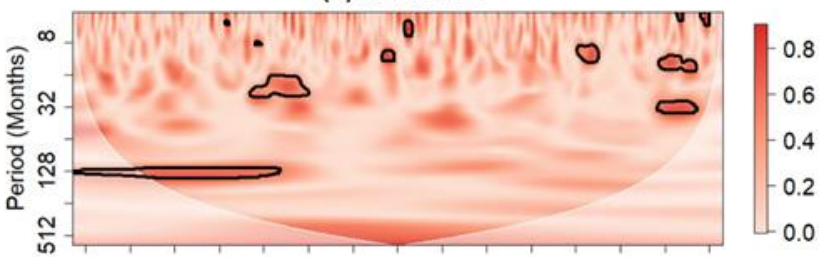

(e) Central Northeast

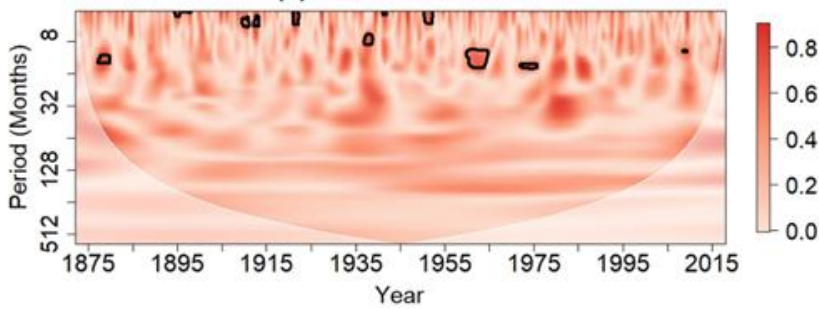

Figure S3. Local diagonal slice of auto-bicoherence spectra corresponding to the (a) All-India, (b) Peninsula, (c) Northwest, (d) Northeast, (e) West Central, and (f) Central Northeast time series. Contours enclose regions of $5 \%$ cumulative arc-wise significance. Light shading represents the cone of influence. 
(a)

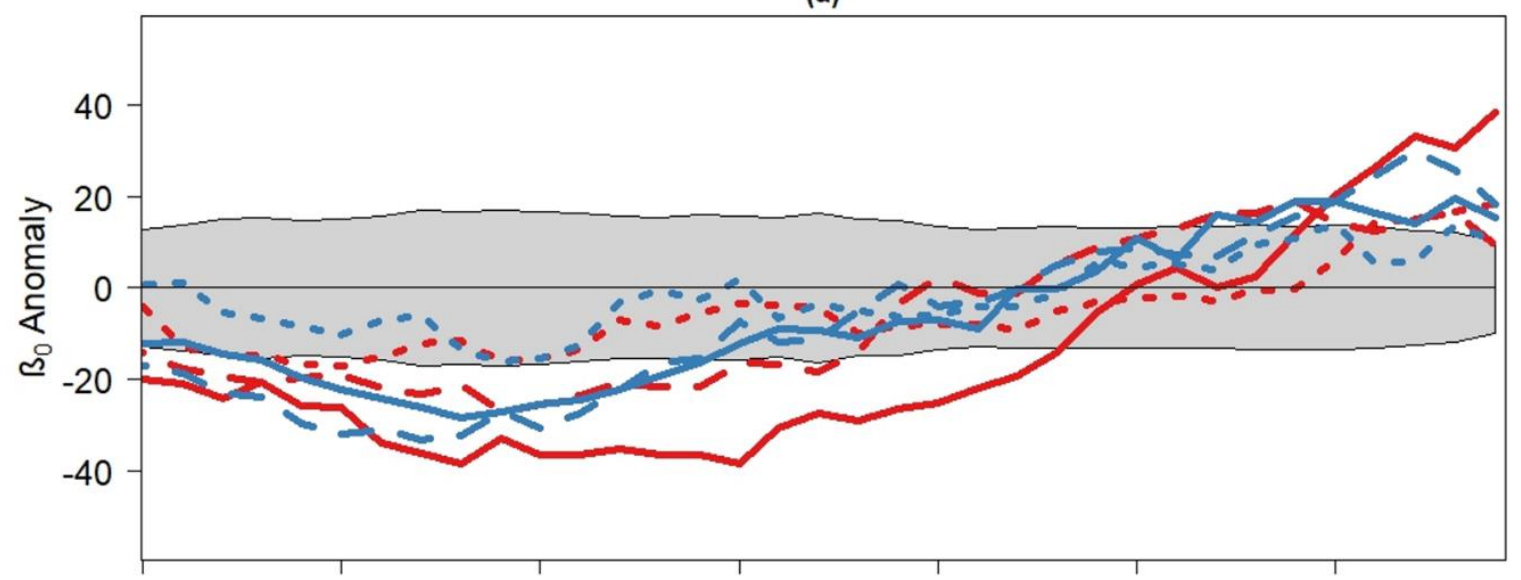

(b)

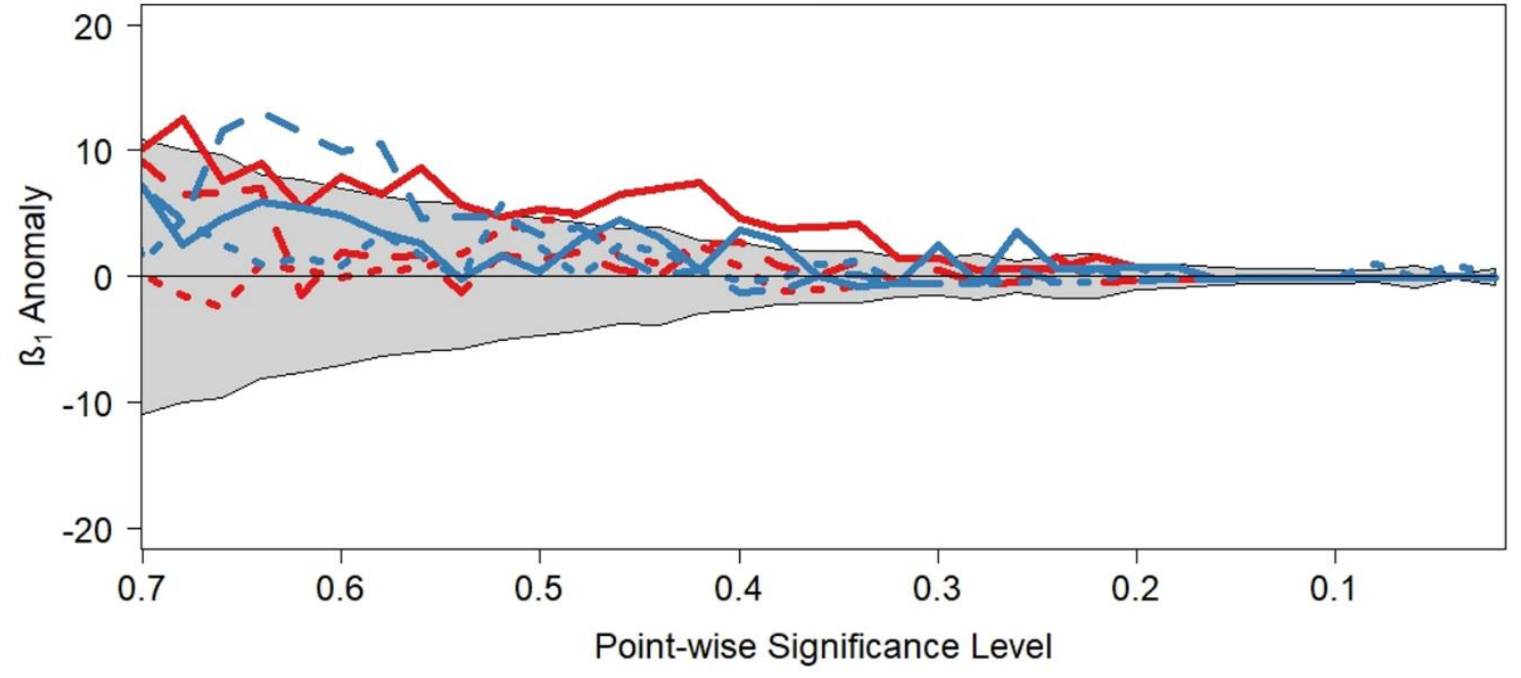

Figure S4. The (a) 0- and (b) 1-dimensional persistent homology profiles (Schulte, 2018) corresponding to the local auto-bicoherence spectra of the India rainfall time series. The grey shaded region is the test nonrejection region, the region in which the null hypothesis of red noise cannot be rejected. 


\section{(e) IOD}

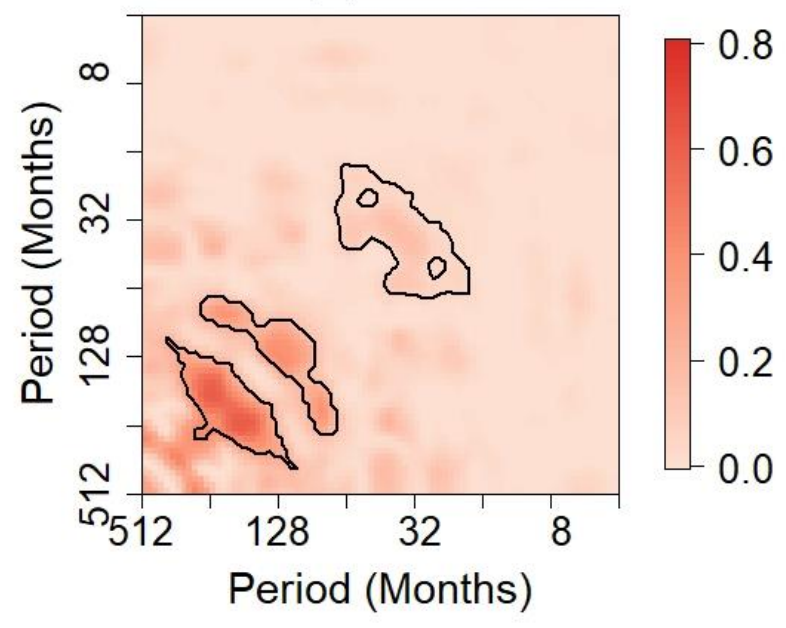

Figure S5. Global auto-bicoherence spectrum of the Indian Ocean dipole index. Contours enclose regions of $5 \%$ cumulative area-wise significance. 


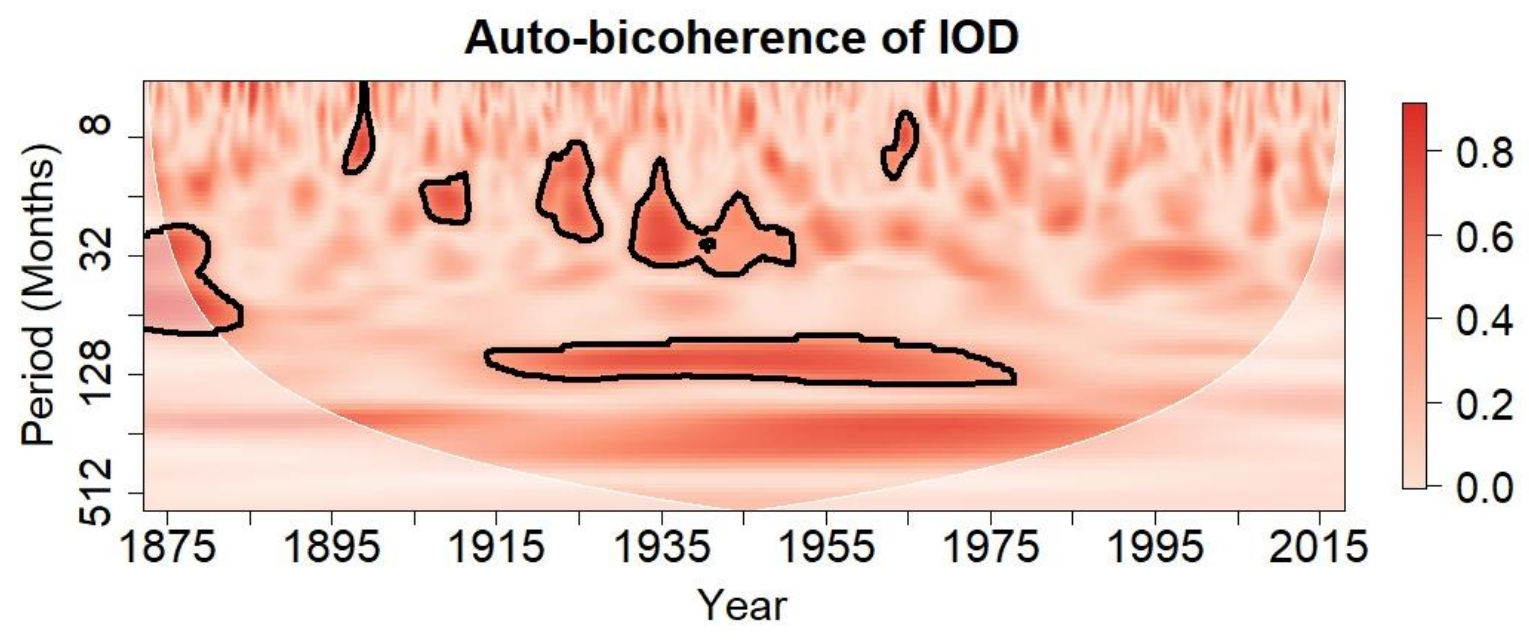

Figure S6. Local auto-bicoherence spectrum of the Indian Ocean dipole index. Contours enclose regions of $5 \%$ cumulative area-wise significance. 


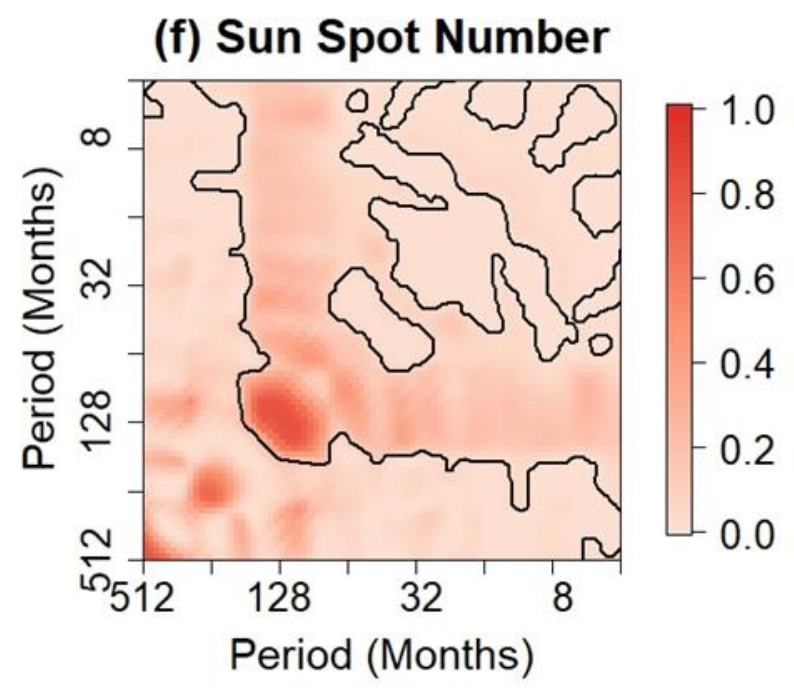

Figure S7. Global auto-bicoherence spectrum of the Sun spot index. Contours enclose regions of 5\% cumulative area-wise significance. 


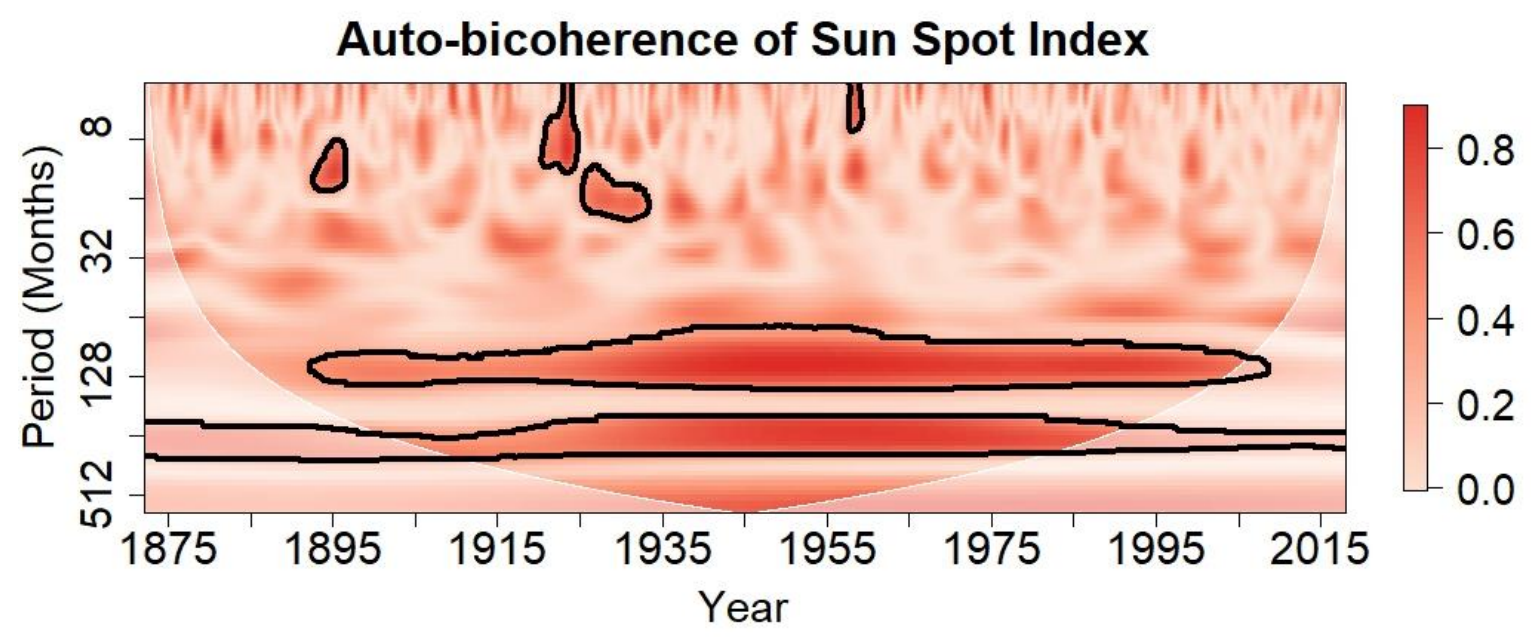

Figure S8. Local auto-bicoherence spectrum of the sun spot index. Contours enclose regions of 5\% cumulative area-wise significance. 